\begin{tabular}{|c|c|}
\hline Title & A erodynamic heating of inflatable aeroshell in orbital reentry \\
\hline Author(s) & Takahashi, Y usuke; Y amada, Kazuhiko \\
\hline Citation & $\begin{array}{l}\text { A cta astronautica, 152, 437-448 } \\
\text { https://doi.org/10.1016/.actaastro.2018.08.003 }\end{array}$ \\
\hline Issue Date & 201811 \\
\hline Doc URL & http:/hdl .handle.net/2115/79647 \\
\hline Rights & $\begin{array}{l}\text { @ 2018. This manuscript version is made available under the CC-BY-NC-ND } 4.0 \text { license } \\
\text { http://creativecommons.org/icenses/by-nc-nd/4.0/ }\end{array}$ \\
\hline Rights(URL) & http://creativecommons.org/icenses/by-nc-nd/4.0/ \\
\hline Type & article (author version) \\
\hline File Information & paper_titans_heatflux.pdf \\
\hline
\end{tabular}

Instructions for use 


\title{
Aerodynamic Heating of Inflatable Aeroshell in Orbital Reentry
}

\author{
Yusuke Takahashi ${ }^{1}$, \\ Hokkaido University, Kita 13 Nishi 8, Kita-ku, Sapporo, Hokkaido 060-8628, Japan \\ and \\ Kazuhiko Yamada ${ }^{2}$ \\ Japan Aerospace Exploration Agency, 3-1-1 Yoshinodai Chuo-ku, Sagamihara, Kanagawa \\ 252-5210, Japan
}

Keywords: Inflatable reentry vehicle, Aerodynamic heating, Hypersonic, Deformation, Coupled analysis

\begin{abstract}
The aerodynamic heating of an inflatable reentry vehicle, which is one of the innovative reentry technologies, was numerically investigated using a tightly coupled approach involving computational fluid dynamics and structure analysis. The fundamentals of a high-enthalpy flow around the inflatable reentry vehicle were clarified. It was found that the flow fields in the shock layer formed in front of the vehicle were strongly in a chemical nonequilibrium state owing to its low-ballistic coefficient trajectory. The heat flux tendencies on the surface of the vehicle were comprehensively investigated for various effects of the vehicle shape, surface catalysis, and turbulence via a parametric study of these parameters. In addition, based on the present results of the computational approach, a new heating-rate method was developed to calculate the heat flux of the nonequilibrium flow. It was demonstrated that the method could well-reproduce the heat flux on the inflatable reentry vehicle.
\end{abstract}

\section{Nomenclature}

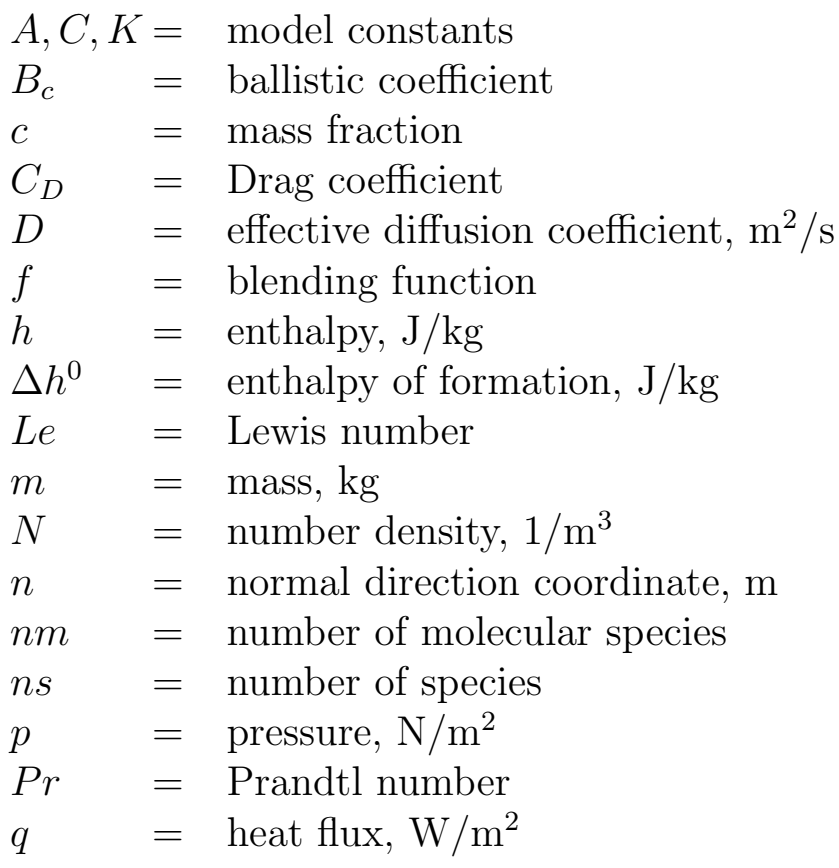

\footnotetext{
${ }^{1}$ Assistant Professor, Faculty of Engineering; ytakahashi@eng.hokudai.ac.jp.

${ }^{2}$ Associate Professor, Institute of Space and Astronautical Science.
} 


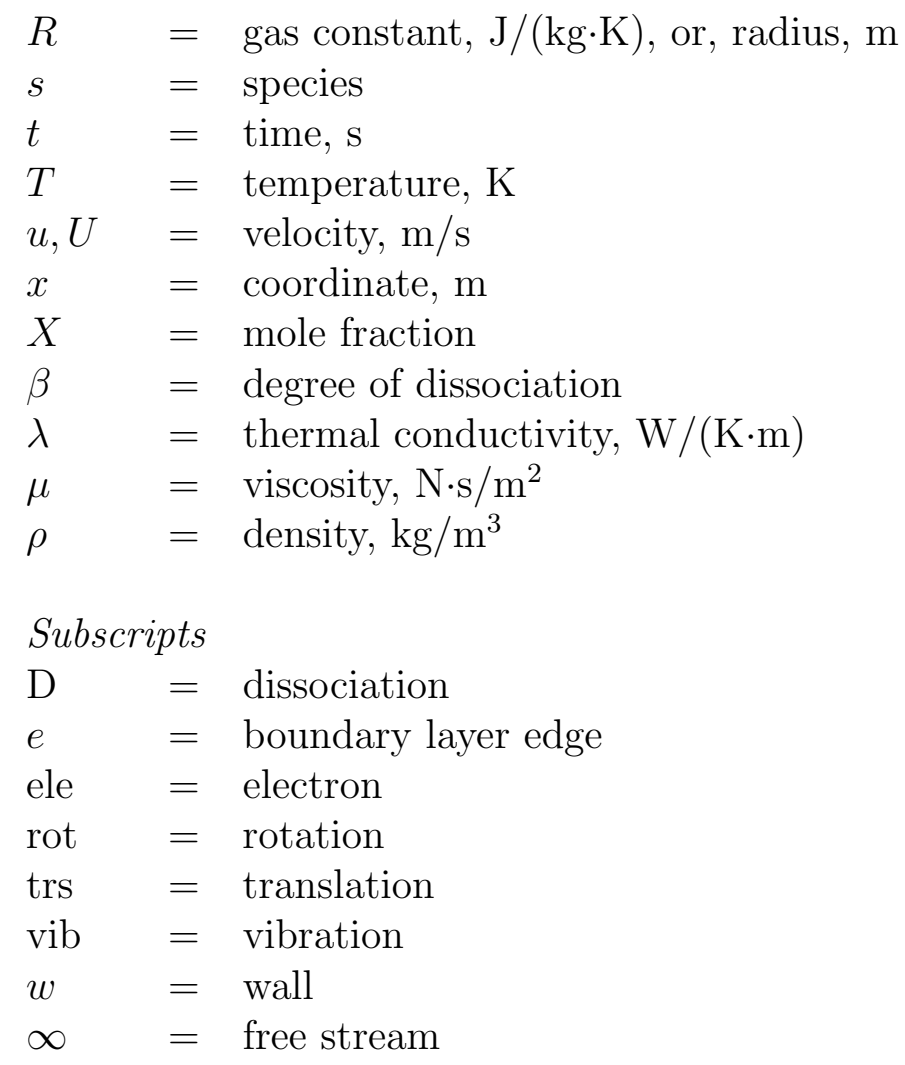

\section{Introduction}

An inflatable aeroshell is being focused on as one of the future reentry technologies. Because of the achievement of a low-ballistic coefficient flight owing to the large area and light weight of the aeroshell, it can offer several advantages such as reduction in the aerodynamic heating $[1,2]$.

Till date, flare-type thin-membrane aeroshells have been developed as part of the Membrane Aeroshell for Atmospheric-entry Capsule (MAAC) Project [3-5]. A suborbital reentry demonstration (Sounding Rocket Experiment of MAAC, SMAAC) was conducted in August 2012 at the Uchinoura Space Center, Kagoshima, Japan [6-8]. This demonstration mission clarified the low-ballistic coefficient flight for a type of inflatable vehicle during reentry. The flight and reentry demonstration of a nano-satellite EGG (re-entry satellite with Gossamer aeroshell and GPS/iridium) [9] in the low-Earth orbit was initiated in January 2017 and successfully completed in May 2017. The EGG was designed to be burned out during atmospheric reentry from the viewpoint of ground safety against falling objects. The temperature history data of the EGG surface was measured above an altitude of approximately $100 \mathrm{~km}$ throughout this mission. Such temperature data are useful to deduce the aerodynamic heating environment during the early phase of reentry for inflatable aeroshells. The EGG did not have a propulsion system to deorbit and to change the attitude. Thus, a controlled reentry using a propulsion system was not realized.

An Earth reentry demonstration mission using an inflatable aeroshell (Titans) [10] including a controlled deorbit achieved by a propulsion device has been proposed. In this mission, the Titans vehicle will deorbit at an altitude of $400 \mathrm{~km}$, and subsequently, start 
the atmospheric reentry. Moreover, after descending in the Earth's atmosphere using an aeroshell instead of a parachute, the vehicle will finally land or splash down. It was indicated in Ref. [11] that Titans can mitigate radio frequency blackout as well as the reduction in the aerodynamic heating.

The heat flux on the surface of the vehicle during reentry is one of the most important design parameters in the development of the Titans vehicle. There have been strong demands for investigating the relation between the shape of the inflatable aeroshell and aerodynamic heating. However, to investigate aerodynamic heating, using only ground-based facilities, such as hypersonic wind tunnels and plasma wind tunnels, is inadequate. This is because these facilities cannot perfectly reproduce the reentry environment. In addition, because usually, an inflatable aeroshell has a flexible membrane, the effect of its deformation needs to be considered for evaluating the aerodynamic heating. However, when considering structure deformation, the similarity of flows as represented by the Mach number and Reynolds number for the wind tunnel testing cannot be applied.

Computational science approach, e.g., computational fluid dynamics (CFD), has become an effective tool for such applications. Aerodynamic simulations for SMAAC [12] and the Hypersonic Inflatable Aerodynamic Decelerator (HIAD) [13] were performed with structure deformation methods. In contrast, studies on the aerodynamic heating analysis are few. In this study, the aerodynamic heating for the Titans vehicle during reentry into the Earth's atmosphere is investigated for various shapes using CFD and structure analysis. In addition, based on the present computational results, a new method to calculate the heat flux on the inflatable reentry surface without a high computational cost is proposed.

\section{Titans Vehicle}

The Titans vehicle mainly consists of three components: a capsule, a membrane aeroshell, and an inflatable torus. Figure 1 shows the schematics of Titans. In the figure, the total length of the Titans vehicle is denoted by $D$. The head capsule with nose radius $R_{\mathrm{c}}$ has a semispherical configuration. The membrane aeroshell with length $L_{\mathrm{m}}$ has a flare angle of $\theta$ and is connected to the inflatable torus. The inflatable torus has a tube with radius $R_{\mathrm{t}}$. In the baseline case, $D, R_{\mathrm{c}}, R_{\mathrm{t}}$, and $\theta$ are $2,500,400,75 \mathrm{~mm}$, and 70 degree, respectively.

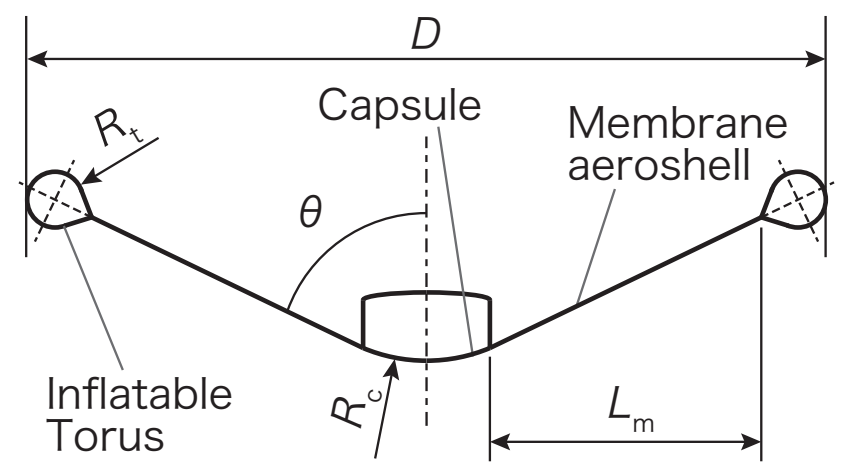

Figure 1: Shape of the Titans vehicle.

Figure 2 shows the reentry trajectory profile of the reentry velocity versus the altitude. This figure also depicts the stagnation heat flux on the Titans vehicle obtained with Tauber's 
model, which is given in units of $\mathrm{W} / \mathrm{m}^{2}$ as

$$
q_{w, \text { tauber }}=1.35 \times 10^{-4} \sqrt{\frac{\rho_{\infty}}{R_{n}}} U_{\infty}^{3.04}\left(1-\frac{h_{w}}{h}\right),
$$

where $R_{n}$ is the nose radius, and $R_{n}=R_{\mathrm{c}}$ in this calculation. Using free stream velocity $U_{\infty}$ and specific heat at constant pressure $C_{p}$, specific flow enthalpy $h$ and specific wall enthalpy $h_{\mathrm{w}}$ are given by $h=\frac{1}{2} U_{\infty}^{2}$ and $h_{\mathrm{w}}=C_{p} T_{\mathrm{w}}$, respectively. The trajectory is determined by solving the equation of motion for the reentry orbit input in the atmosphere model and using the ballistic coefficient, which is defined as

$$
B_{\mathrm{c}}=\frac{m}{S C_{\mathrm{D}}},
$$

where $m$ and $S$ are the mass of the vehicle and front-projected area, respectively. The drag coefficient, $C_{\mathrm{D}}$, is 1.5 at supersonic speed and 1.0 at subsonic speed. The vehicle deorbits from an initial circular orbit having an altitude of $400 \mathrm{~km}$ with a delta velocity of $90 \mathrm{~m} / \mathrm{s}$.

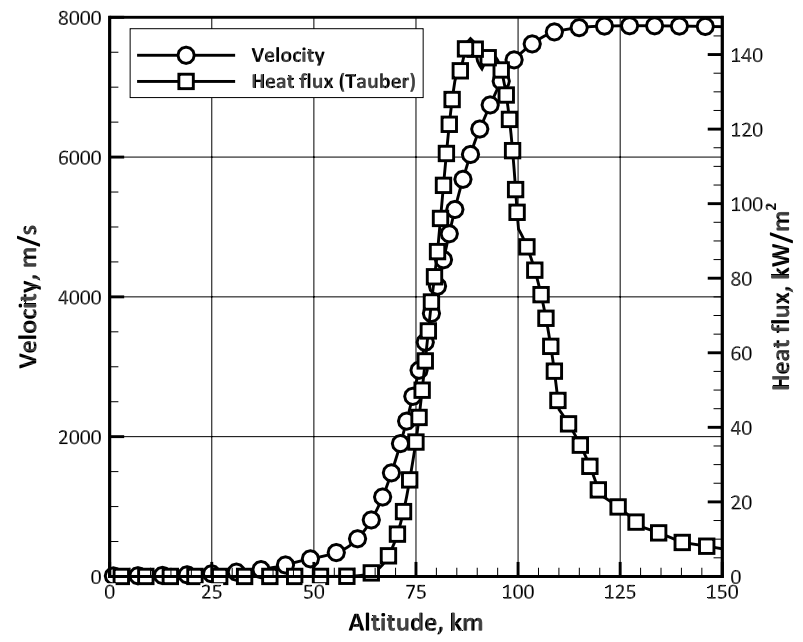

Figure 2: Reentry trajectory of the Titans vehicle.

\section{$3 \quad$ Numerical Method}

\subsection{Modeling}

For the flow field simulation, except for the deformation model, the governing equations and physical models used here are basically the same as those used in the study by Takahashi [11]. The present analysis model for a hypersonic speed was validated by the numerical simulations of the reentry flow around the Hayabusa sample return capsule [14].

The program code used herein assumes that the flow is laminar, steady, and continuum; flow field is in a thermochemical nonequilibrium; and temperature is separated into translational, rotational, vibrational, and electron temperatures. The governing equations are composed of the total mass, momentum, total energy, species mass, rotation energy, vibration energy, and electron energy conservations, in addition to the equation of state. 
The transport properties such as viscosity, thermal conductivity, and binary diffusion coefficients for a mixture gas are evaluated using Yos' formula [15], which is based on the first-order Chapman-Enskog approximation. The collision cross-sections are obtained using Gupta's method [16]. For e-N and e-O pairs, the collision cross-section models of Fertig et al. $[17,18]$ are used. The diffusion coefficients are expressed using the formula developed by Curtiss and Hirschfelder [19]. An ambipolar diffusion is assumed for the charged species.

For modeling the chemical reactions occurring in high-temperature air, the gas is assumed to consist of 11 chemical species, namely, $\mathrm{N}_{2}, \mathrm{O}_{2}, \mathrm{NO}, \mathrm{N}_{2}^{+}, \mathrm{O}_{2}^{+}, \mathrm{NO}^{+}, \mathrm{N}, \mathrm{O}, \mathrm{N}^{+}, \mathrm{O}^{+}$, and $\mathrm{e}^{-}$, and 49 reactions are assumed to occur. The mass production rates are calculated using the forward and backward reaction rates of the chemical reactions. The forward reaction rate coefficients are obtained from Park's work [20]. Moreover, the backward reaction rate is evaluated using the corresponding equilibrium constant. The equilibrium constants for the chemical reactions are calculated using the curve-fit formula and the parameters are obtained with Park's model [21] and Gupta's model [16].

The present analysis model introduces energy transfer between the internal energy modes: translation-rotation, translation-vibration, translation-electron, rotation-vibration, rotationelectron, and vibration-electron. The energy loss and release for the vibrations and rotations associated with heavy-particle impact reactions are obtained using a non-preferential dissociation model. The electron energy loss and release due to the electron-impact dissociation and ionization are also considered.

To express the deformation of the membrane aeroshell in terms of the aerodynamic force, a membrane deformation model using a particle spring-mass system [12] is coupled with the flow field simulation.

\subsection{Numerical Procedure}

The governing equations of the flow field are solved using a finite volume approach. In addition, the equation system is transformed into the delta form to obtain a steady-state solution, which is updated at each time step. All the flow field properties are set at the center of a control volume in a computational domain. The advection fluxes in the equations are calculated using the SLAU scheme [22] with the MUSCL interpolation method. The viscous fluxes are evaluated using the central difference method. Thus, the spatial accuracy achieves the second order. For time integration, we employ the lower-upper symmetric GaussSeidel (LU-SGS) method [23] coupled with the point-implicit method [24]. The deformation calculation is performed at every 5,000 iterations of the flow field calculation. These models are implemented in "Arcflow/Arcwave" code.

\subsection{Boundary Conditions}

The computational boundaries are composed of the inflow boundary, outflow boundary, axisymmetric boundary on the center axis, and surface boundary on the vehicle surface. At the inflow boundary, the free stream conditions listed in Table 1 based on the reentry trajectory are applied. The chemical composition of the inflow is 0.765 of $\mathrm{N}_{2}$ and 0.235 of $\mathrm{O}_{2}$ in mass fractions. A gradient-free condition for all the flow parameters is imposed at the outflow boundary. A non-slip condition for the velocity and condition of no pressure gradient normal to the wall are imposed at the surface boundary. Temperature $T_{\mathrm{w}}$ is fixed as $300 \mathrm{~K}$ at the surface. In addition, the surface is assumed to be a non-catalytic wall. 
Table 1: Free stream parameters along the Titans reentry trajectory.

\begin{tabular}{c|ccc}
\hline \hline Altitude, $\mathrm{km}$ & Velocity, $\mathrm{m} / \mathrm{s}$ & Density, $\mathrm{kg} / \mathrm{m}^{3}$ & Temperature, $\mathrm{K}$ \\
\hline 108 & 7,773 & $1.889 \times 10^{-7}$ & 231.3 \\
103 & 7,605 & $4.142 \times 10^{-7}$ & 209.4 \\
98 & 7,363 & $9.014 \times 10^{-7}$ & 194.0 \\
93 & 6,809 & $2.181 \times 10^{-6}$ & 189.5 \\
88 & 6,038 & $4.858 \times 10^{-6}$ & 187.0 \\
83 & 5,081 & $1.105 \times 10^{-5}$ & 191.2 \\
79 & 3,763 & $2.358 \times 10^{-5}$ & 201.1 \\
73 & 2,396 & $5.125 \times 10^{-5}$ & 211.5 \\
\hline \hline
\end{tabular}

The computational grids of the initial and deformed shapes for the baseline case of the Titans vehicle at an altitude of $88 \mathrm{~km}$ are shown in Figs. 3(a) and 3(b), respectively. The deformed grids are obtained by the deformation calculation, starting from the initial grids. It should be noted that separate initial grid systems are used for each altitude condition. Structured grid systems are used for each analysis. The number of computational grids is $100 \times 95$ (axial $\times$ radial) for this case.

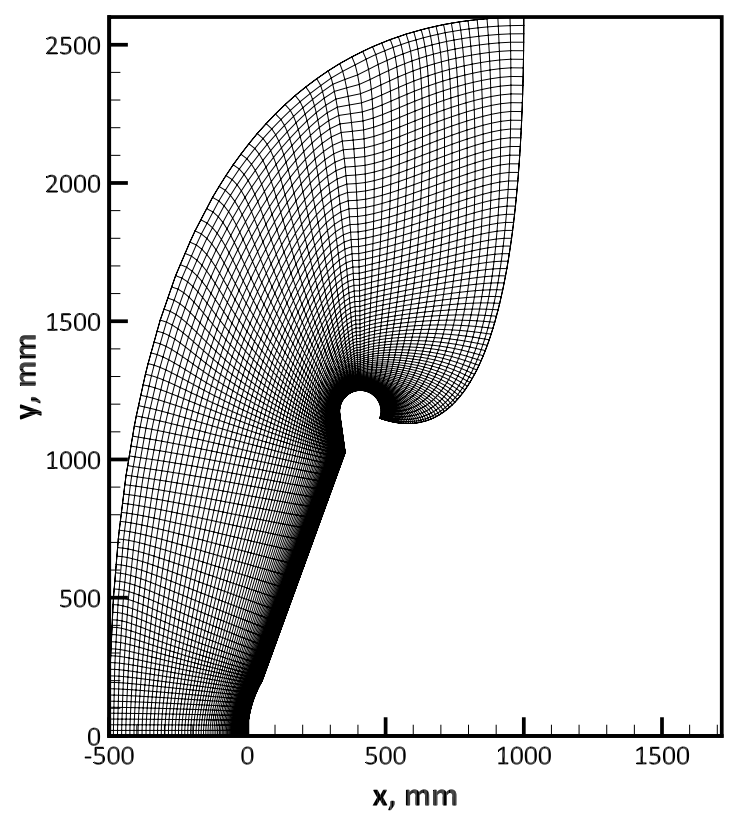

(a) Initial (rigid) shape.

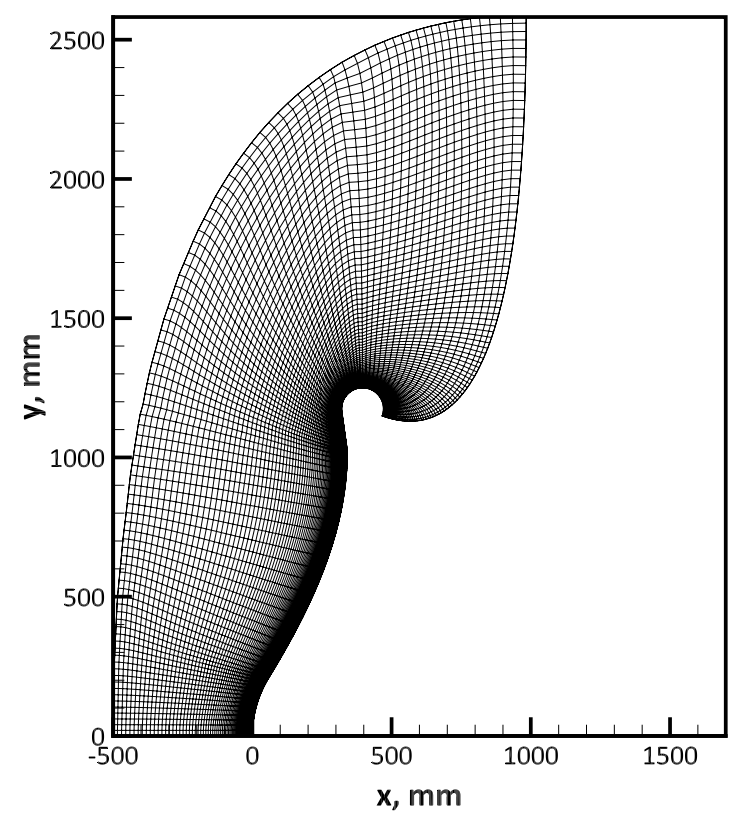

(b) Example of a deformed shape owing to membrane deformation.

Figure 3: Computational grids for the Titans vehicle shape in the baseline case at an altitude of $88 \mathrm{~km}$. 


\subsection{Parameter Study}

To investigate the heat flux tendency on the surface during atmospheric reentry, a parametric study is performed for the Titans vehicle. The dependency on shape parameters such as the diameter, nose radius of the head capsule, and tube radius of the torus is examined. In addition, in this study, the flare angle of the membrane, Young's modulus of the deformation model, turbulence, and wall catalysis are considered as variable parameters. Calculation parameters for the parametric study are listed in Table 2.

Table 2: Calculation parameters.

\begin{tabular}{l|cccccccc}
\hline \hline Case & $D, \mathrm{~mm}$ & $R_{c}, \mathrm{~mm}$ & $R_{t}, \mathrm{~mm}$ & $L_{m}, \mathrm{~mm}$ & $\theta,^{\circ}$ & $E, \mathrm{~Pa}$ & $\gamma$ & Flow \\
\hline TS1 & 1,500 & 400 & 45 & 415 & 70 & $1.0 \times 10^{9}$ & 0 & Laminar \\
TS2 & 2,500 & 400 & 75 & 825 & 70 & $1.0 \times 10^{9}$ & 0 & Laminar \\
TS3 & 3,500 & 400 & 105 & 1235 & 70 & $1.0 \times 10^{9}$ & 0 & Laminar \\
TS4 & 5,000 & 400 & 150 & 1850 & 70 & $1.0 \times 10^{9}$ & 0 & Laminar \\
TS5 & 5,000 & 800 & 150 & 1650 & 70 & $1.0 \times 10^{9}$ & 0 & Laminar \\
TS2_F65 & 2,500 & 400 & 75 & 825 & 65 & $1.0 \times 10^{9}$ & 0 & Laminar \\
TS2_F75 & 2,500 & 400 & 75 & 825 & 75 & $1.0 \times 10^{9}$ & 0 & Laminar \\
TS2_E1e8 & 2,500 & 400 & 75 & 825 & 70 & $1.0 \times 10^{8}$ & 0 & Laminar \\
TS2_E3e7 & 2,500 & 400 & 75 & 825 & 70 & $3.0 \times 10^{7}$ & 0 & Laminar \\
TS2_Rigid & 2,500 & 400 & 75 & 825 & 70 & $-(E \rightarrow \infty)$ & 0 & Laminar \\
TS2_FCW & 2,500 & 400 & 75 & 825 & 70 & $1.0 \times 10^{9}$ & 1 & Laminar \\
TS2_Turb & 2,500 & 400 & 75 & 825 & 70 & $1.0 \times 10^{9}$ & 0 & Turbulent \\
\hline \hline
\end{tabular}

Shape, in particular, dimension, is one of the most important parameters that directly affects the aerodynamic deceleration performance and mass of an inflatable reentry vehicle. Cases of the dimension parameters of the Titans vehicle shape are "TS1", "TS2", "TS3", "TS4", and "TS5". Diameter $D$ and tube radius of the torus $R_{t}$ are similar in these cases. In the table, "TS2" represents the baseline case mentioned previously.

The dependency on the flare angle of the membrane is examined because it is expected to affect the heat flux on the surface. The parameters of the flare angle, $\theta$, used herein are $65^{\circ}$ for "TS2_F65" and $75^{\circ}$ for "TS2_F75," in addition to $70^{\circ}$ for "TS2".

In the present membrane deformation model, the deformation is determined by the relation between the elastic force of the membrane and the aerodynamic force. The elastic force, which is composed of tension force and bending force, is linear with the Young's modulus, which is a characteristic parameter of the membrane deformation. The effect of deformation on the heat flux is investigated by varying the Young's modulus, $E$, of $1.0 \times 10^{9}$ for "TS2" case, $1.0 \times 10^{8}$ for "TS2_E1e8", and $3.0 \times 10^{7}$ for "TS2_E3e7". In addition, a rigid case assuming $E \rightarrow \infty$ for "TS2_Rigid" is investigated.

Because the catalysis of the material (Zylon) of the membrane and inflatable torus is unclear, it is necessary to investigate its effect on the heat flux. The catalytic effect on the Titans vehicle surface is defined by the boundary condition, which is expressed as

$$
\rho D_{s} \frac{\partial C_{s}}{\partial n}=\gamma \sqrt{\frac{R_{s} T_{w}}{2 \pi}} \rho_{s}
$$


where $D_{s}, C_{s}, M_{s}, R_{s}$, and $n$ are the effective diffusion coefficient of species $s$, mass fraction, molar mass, gas constant, and coordinate in the normal direction to the wall surface, respectively. When catalytic coefficient $\gamma=0$ and $\gamma=1$, the surface boundary conditions are defined as a non-catalytic wall (NCW) for "TS2" and a fully catalytic wall (FCW) for "TS2_FCW", respectively.

The turbulence effect is also investigated using the models for "TS2_Turb" case. The shear stress transport model (1994 version) [25] is adopted as the turbulent model.

\section{Results and Discussion}

In this section, first, the fundamental structure of a high-enthalpy flow for the Titans vehicle in the baseline case (hereafter referred as Titans "TS2" case) obtained by the present analysis model is described. Subsequently, the heat flux distribution on the Titans vehicle surface and results computed by the parametric study are discussed. Finally, a method to evaluate the aerodynamic heating on an inflatable reentry vehicle is proposed based on the results obtained herein.

\subsection{General}

Figures 4(a) and 4(b) show the axial profiles of the translational temperature and degree of dissociation for the Titans "TS2" case at various altitudes, respectively. The degree of dissociation $(\beta)$ in this high-temperature air system is calculated by

$$
\beta=\frac{0.5\left(N_{\mathrm{N}}+N_{\mathrm{O}}+N_{\mathrm{N}^{+}}+N_{\mathrm{O}^{+}}\right)}{N_{\mathrm{N}_{2}}+N_{\mathrm{O}_{2}}+N_{\mathrm{NO}}+N_{\mathrm{N}_{2}^{+}}+N_{\mathrm{O}_{2}^{+}}+N_{\mathrm{NO}^{+}}+0.5\left(N_{\mathrm{N}}+N_{\mathrm{O}}+N_{\mathrm{N}^{+}}+N_{\mathrm{O}^{+}}\right)},
$$

where $N_{s}$ is the number density of the chemical species.

Owing to the high-velocity reentry, a strong shock wave is formed in front of the vehicle. It is confirmed that the translational temperature increases across the shock wave and a high-temperature region is formed. At higher altitudes, the chemical reactions proceed at a slow speed in the shock layer, which causes the translational temperature to increase. This is because of the low frequency of collisions between the molecules owing to the low-density environment. In contrast, at a lower altitude, e.g., $73 \mathrm{~km}$, an equilibrium region appears behind the shock wave, although chemical reactions barely occur. For the Titans cases, molecular dissociations, and not ionizations, are the dominant reactions in the shock layer. The pressure is uniformly constant from the capsule head to the inflatable torus, although the distribution is not shown here. The membrane aeroshell is deformed by the aerodynamic force, and consequently, the effective flare angle becomes slightly small. When the effective flare angle is less than a certain value, a transition from a normal shock wave to a detached shock wave can occur over the head capsule and subsequently, locally increase the heat flux. The formation of an actual locally detached shock wave is confirmed for the cases with varying flare angle and Young's modulus parameters, as will be mentioned later.

The heat flux distribution on the vehicle surface in the Titans "TS2" case at different altitudes is displayed in Fig. 5. The heat flux is obtained using the following expression:

$$
q_{j}=\lambda_{\mathrm{trs}} \frac{\partial T_{\mathrm{trs}}}{\partial x_{j}}+\lambda_{\mathrm{rot}} \frac{\partial T_{\mathrm{rot}}}{\partial x_{j}}+\lambda_{\mathrm{vib}} \frac{\partial T_{\mathrm{vib}}}{\partial x_{j}}+\lambda_{\mathrm{ele}} \frac{\partial T_{\mathrm{ele}}}{\partial x_{j}}+\rho \sum_{s=1}^{n s} h_{s} D_{s} \frac{\partial X_{s}}{\partial x_{j}} .
$$




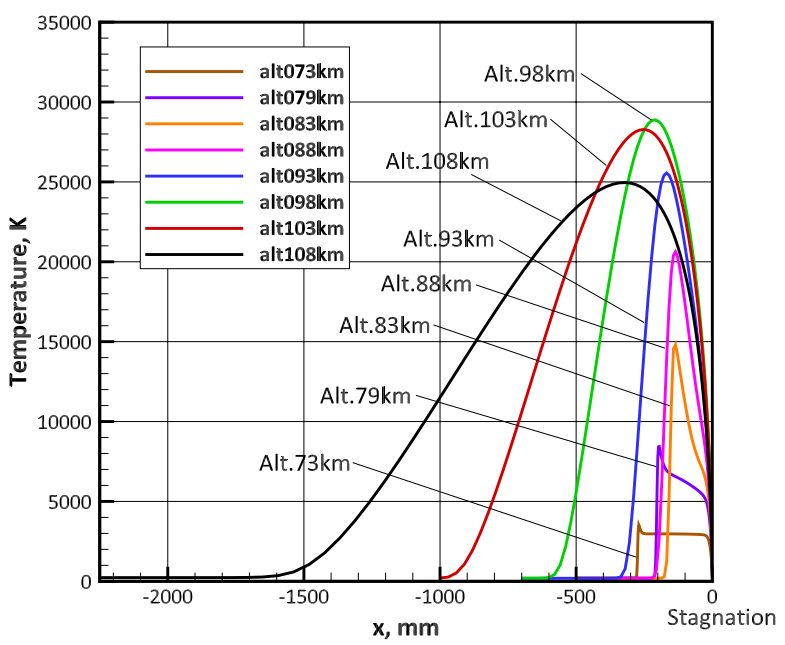

(a) Translational temperature.

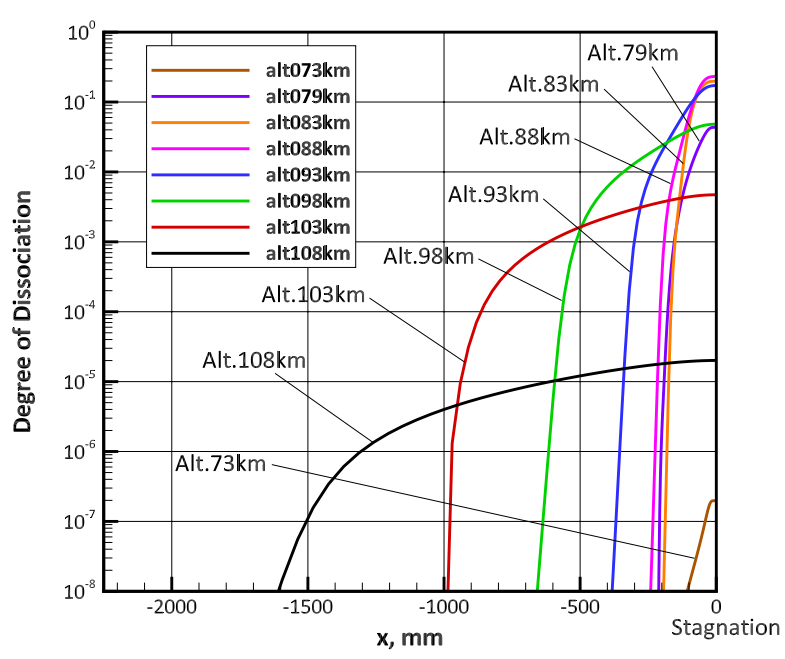

(b) Degree of dissociation.

Figure 4: Profiles of the translational temperature and degree of dissociation along the center axis for the Titans "TS2" case at various altitudes.

Because the non-catalytic wall condition is imposed for baseline case "TS2", in the above equation, the fifth term on the right-hand side is zero on the surface. At each altitude, the heat flux exhibits a peak value on the capsule top and continues to decrease toward the inflatable torus. Subsequently, the heat flux reaches again high values on the inflatable torus. The peak value on the capsule head is approximately $102 \mathrm{~kW} / \mathrm{m}^{2}$, which is low compared with that of Tauber's model, which is approximately $124 \mathrm{~kW} / \mathrm{m}^{2}$, at an altitude of $98 \mathrm{~km}$. This is because the selected effective nose radius is different. Tauber's model can be applied to a sphere-shaped model, using its nose radius. However, the inflatable reentry vehicle is much different from a sphere shape because of the membrane aeroshell and inflatable torus. The peak heat flux on the inflatable torus is approximately $60 \mathrm{~kW} / \mathrm{m}^{2}$ at an altitude of 103 $\mathrm{km}$. It indicates that the heat flux environment on the inflatable torus is severe at high altitudes.

To investigate the computational grid independency in the present simulations, grid studies were performed for the Titans "TS2" case at an altitude of $83 \mathrm{~km}$. We used fine computational grids with $150 \times 150$ (axial $\times$ radial) points. The relative error in the heat flux at the stagnation point using the present and fine grids was within $0.64 \%$. This result indicates that the present grids are sufficiently converged.

\subsection{Shape Effect}

The shape effect on the heat flux is investigated for the cases "TS1", "TS2", "TS3", "TS4", and "TS5" listed in Table 2. Figures 6(a), 6(b), and 6(c) present the heat flux histories versus the altitude at the capsule, membrane, and inflatable torus for each case, respectively. Here, the positions of the "capsule", "membrane", and "inflatable torus" are defined as the top of the capsule $(y=0)$, near the center of the membrane (defined by $y=R_{\mathrm{c}} / 2+L_{\mathrm{m}} / 2$ ), and peak heat flux point on the torus, respectively. As the diameter of Titans becomes large, the heat flux at these three positions tends to reduce. Thus, a large-scale reentry vehicle has an advantage from the viewpoint of aerodynamic heating. The comparison of the "TS4" and 


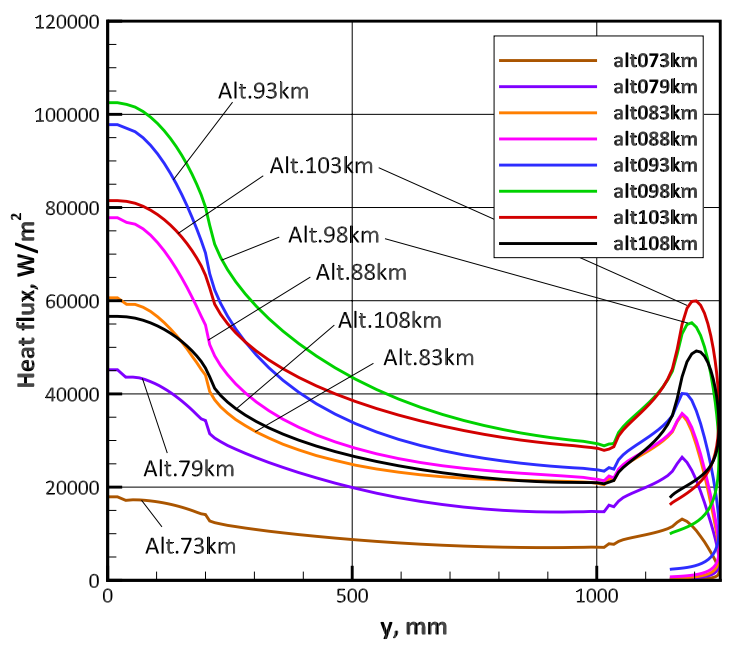

Figure 5: Heat flux on the Titans vehicle surface at various altitudes for baseline case "TS2".

"TS5" cases reveals that the nose radius of the capsule affects the heat flux on the capsule, whereas the nose radius has practically no effect on the heat flux on the membrane and inflatable torus.

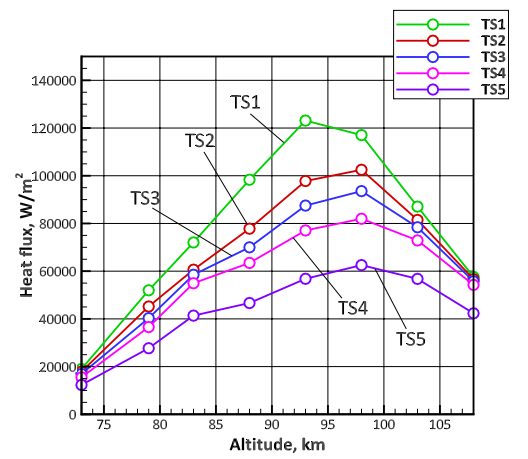

(a) Capsule.

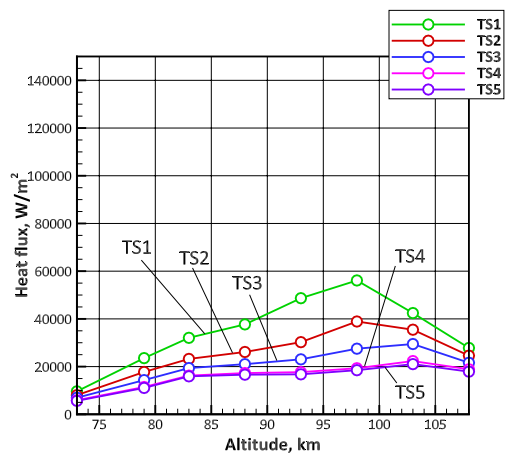

(b) Membrane.

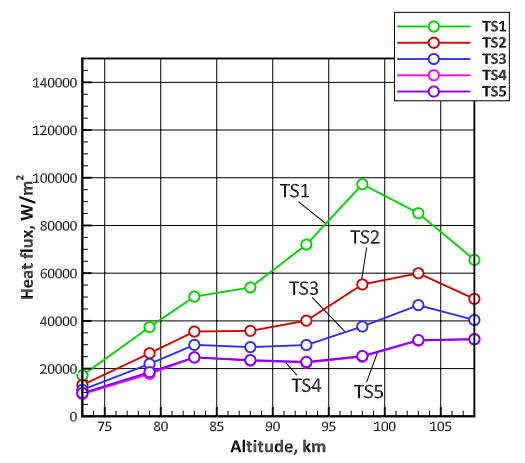

(c) Inflatable torus.

Figure 6: Heat flux histories at the capsule, membrane, and inflatable torus for the "TS1", "TS2", "TS3", "TS4", and "TS5" cases.

\subsection{Flare Angle Effect}

The flare angle of the membrane has a significant effect on the aerodynamic heating as well as the center of the aerodynamic force, i.e., the attitude of the vehicle. Hence, it is important to evaluate its tendency for the heating environment. Figures 7(a), 7(b), and 7(c) display the heat flux histories versus the altitude at the capsule, membrane, and inflatable torus for "TS2", "TS2_F65", and "TS2_F75" cases, respectively. It is found that the flare angle has a relatively small and slight effect on the membrane and inflatable torus, respectively. In contrast, the flare angle has a major impact on the heat flux at the top of the capsule. In particular, a large increase in the stagnation heat flux is confirmed in the low-flare angle case 
("TS2_F65"). Figures 8(a) and 8(b) show the distributions of the translational temperature around the Titans vehicle for flare angles of $65^{\circ}$ and $75^{\circ}$ ("TS2_F65" and "TS2_F75") at an altitude of $88 \mathrm{~km}$, respectively. As confirmed in these distributions, the increase in the heat flux in the "TS2_F65" case is mainly because of the formation of a locally detached shock wave with the increase in the temperature gradient near the capsule.

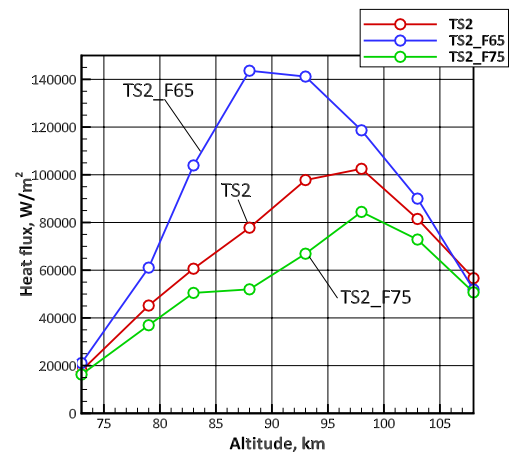

(a) Capsule.

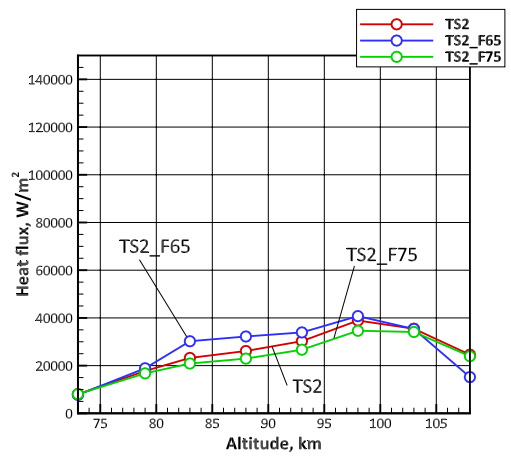

(b) Membrane.

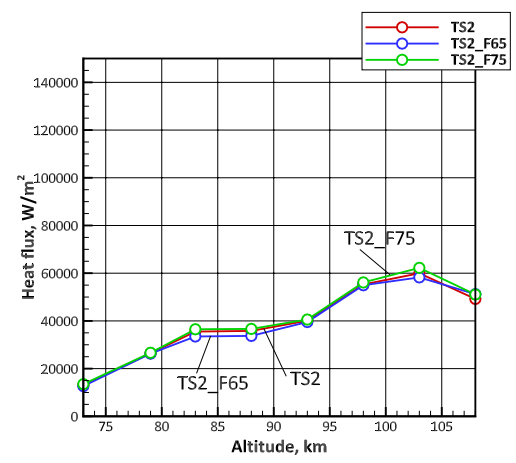

(c) Inflatable torus.

Figure 7: Heat flux histories at the capsule, membrane, and inflatable torus for the "TS2", "TS2_F65", and "TS2_F75" cases.

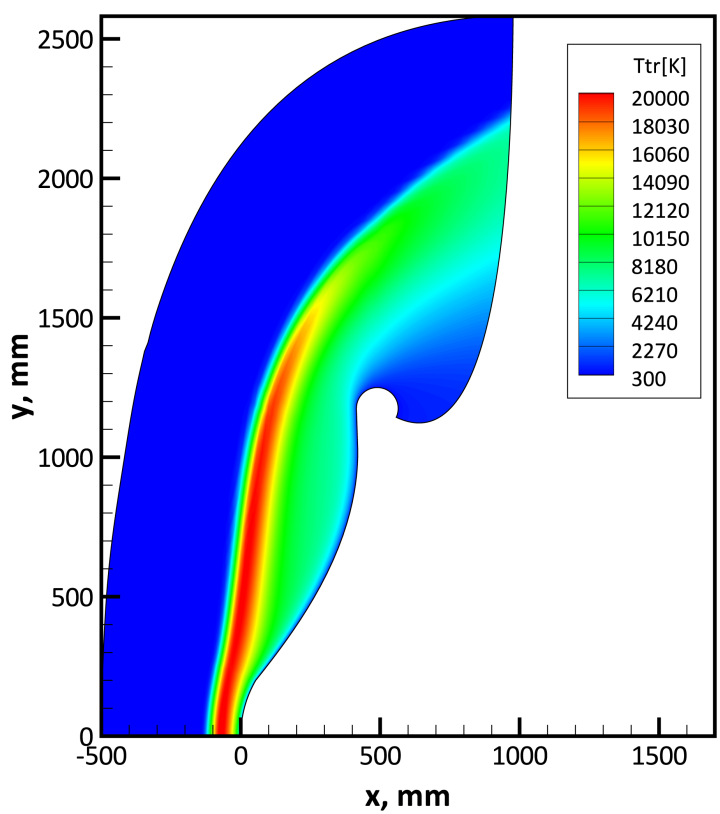

(a) Flare angle of $65^{\circ}$ ("TS2_F65").

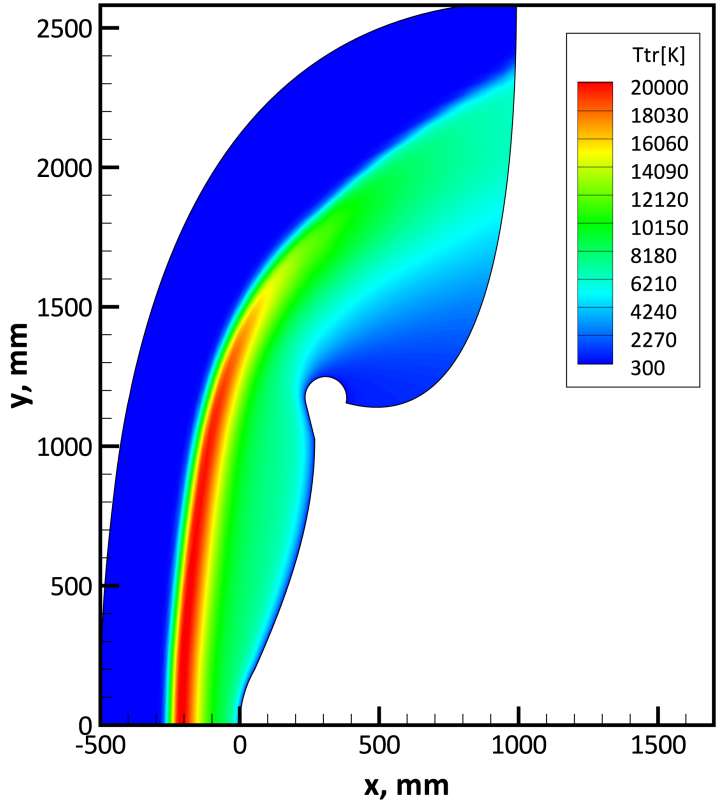

(b) Flare angle of $75^{\circ}$ ("TS2_F 75").

Figure 8: Distribution of the translational temperature around the Titans vehicle for the "TS2_F65" and "TS2_F75" cases at an altitude of $88 \mathrm{~km}$. 


\subsection{Deformation Effect}

Figures 9(a), 9(b), and 9(c) depict the heat flux histories versus the altitude at the capsule, membrane, and inflatable torus, respectively, in the "TS2", "TS2_E1e8", "TS2_E3e7", and "TS2_Rigid" cases. Because the deformation of the membrane when E=30 MPa ("TS2_E3e7" case) is notably large, the shock wave formation in this case is different from the other cases. The membrane and inflatable torus in the "TS2_E3e7" case primarily move back, and a locally detached shock wave is formed over the capsule head. This also occurs in the case where the flare angle is $65^{\circ}$ ("TS2_F65" case). Moreover, the shock stand-off distance becomes short, which causes an increase in the temperature gradient near the capsule surface. This is the main reason for the heat flux at the capsule being high in this case. In the "TS2_E1e8", "TS2", and "TS2_Rigid" cases, the heat flux at the capsule tends to increase as the deformation enlarges. In contrast, it is found that the heat fluxes on the inflatable torus are almost the same in the three cases. This is mainly because the shock stand-off distance over the torus part is practically constant for these cases.

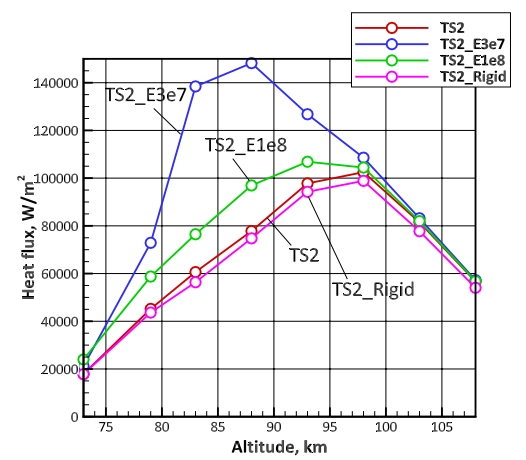

(a) Capsule.

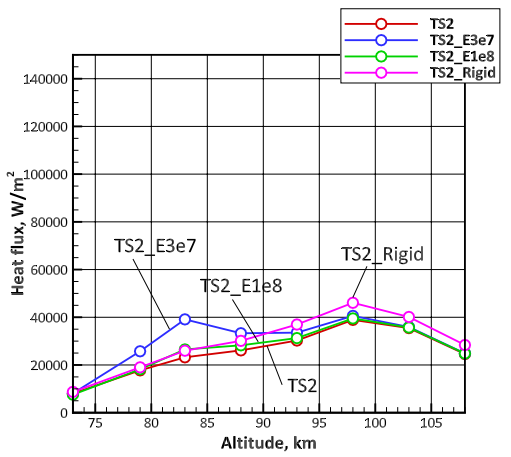

(b) Membrane.

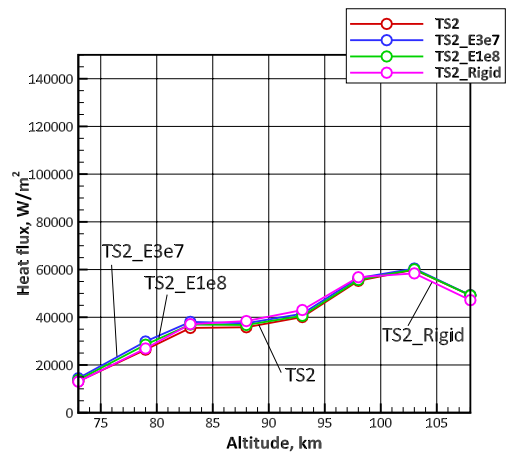

(c) Inflatable torus.

Figure 9: Heat flux histories at the capsule, membrane, and inflatable torus for the "TS2", "TS2_E1e8", "TS2_E3e7", and "TS2_Rigid" cases.

\subsection{Catalysis Effect}

The heat flux histories at the Titans capsule, membrane, and inflatable torus at different altitudes under the NCW and FCW conditions ("TS2" and "TS2_FCW") are shown in Figs. 10(a), 10(b), and 10(c), respectively. The peak heating altitudes in the present CFD approach are $98 \mathrm{~km}$ for NCW and $93 \mathrm{~km}$ for FCW. The FCW condition shows a large increase in the aerodynamic heating at all the positions because of the energy release due to the recombination reactions occurring on the surface. The heat fluxes on the Titans vehicle surface at all the altitudes reach peak values of approximately 102 and $121 \mathrm{~kW} / \mathrm{m}^{2}$ on the stagnation point of the capsule in the NCW and FCW cases, respectively. At higher and lower altitudes, the differences in the heat fluxes in the two cases are small because chemical reactions do not occur in the shock layer. The catalysis effect of Zylon for gas dissociation is not clear, as mentioned earlier. However, it is clarified that the catalysis effect has a significant effect on the heat flux on the surface of the inflatable reentry vehicle. Therefore, it is important to accurately evaluate the heat flux by considering the catalysis for the design and development of this type of reentry vehicle. 


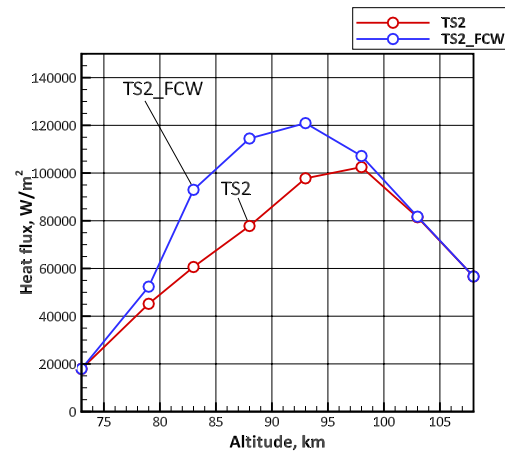

(a) Capsule.

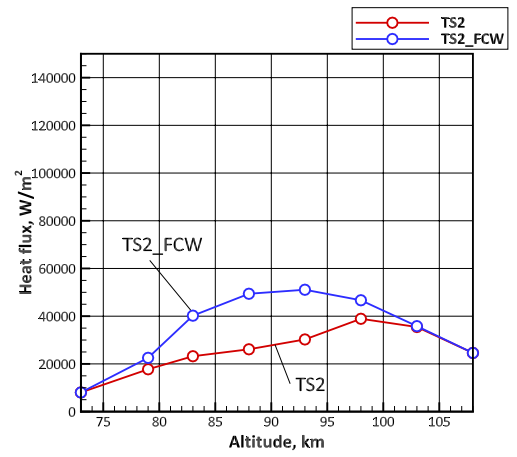

(b) Membrane.

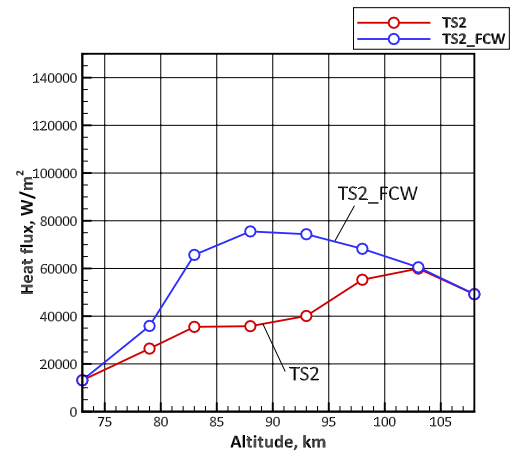

(c) Inflatable torus.

Figure 10: Heat flux histories at the capsule, membrane, and inflatable torus for the "TS2" and "TS2_FCW" cases.

\subsection{Turbulence Effect}

A comparison of the results for the laminar and turbulent cases is presented here. Figures 11(a), 11(b), and 11(c) are the heat flux histories versus the altitude at the capsule, membrane, and inflatable torus, respectively, for the "TS2" and "TS2_Turb" cases. Similar to the case of FCW condition, the heat fluxes at the three positions become high. Owing to the momentum and energy transfers caused by the turbulence mixing in the shock layer, the temperature gradient near the Titans vehicle surface becomes large, which increases the heat flux. At higher altitudes of 108 and $103 \mathrm{~km}$, the discrepancy between the laminar and turbulent cases is small because of the low Reynolds number resulting from the low density.

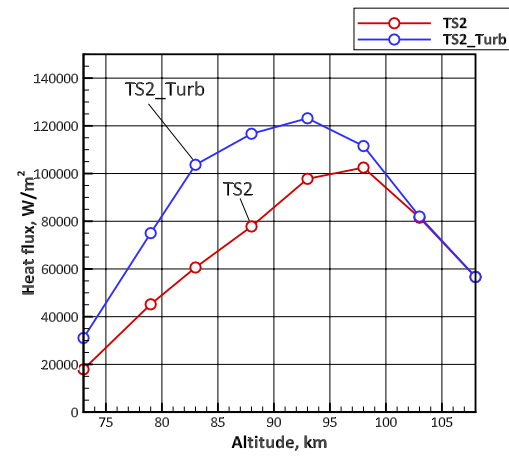

(a) Capsule.

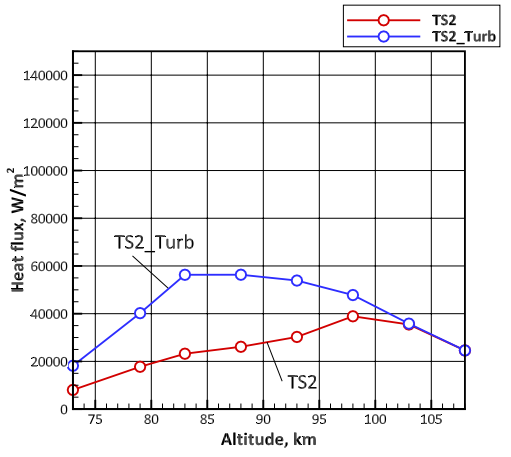

(b) Membrane.

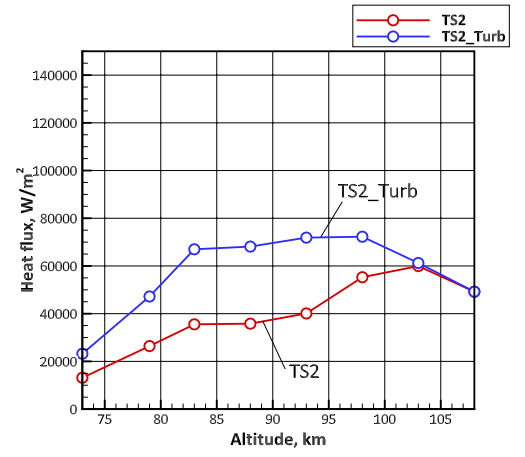

(c) Inflatable torus.

Figure 11: Heat flux histories at the capsule, membrane, and inflatable torus for the "TS2" and "TS2_Turb" cases.

\subsection{Heating-Rate Method}

Based on the present CFD results focusing on the effects of the shape and surface catalysis, we propose a heat flux model for the inflatable reentry vehicle. According to Marvin and Pope [26,27], heat flux at the stagnation point under the FCW condition can be evaluated 
as follows:

$$
q_{\mathrm{c}, \mathrm{fcw}} \sqrt{\frac{R}{p_{0}}}=K\left(h-h_{w}\right)
$$

Under the NCW condition, assuming that the Lewis number is unity,

$$
q_{\mathrm{c}, \mathrm{ncw}} \sqrt{\frac{R}{p_{0}}}=K\left(h-h_{w}\right)\left(1-\frac{c \Delta h^{0}}{h}\right)
$$

where $K$ and $p_{0}$ are the constant and dynamic pressure, respectively. In addition, $\Delta h^{0}$ denotes the enthalpy of formation, and, $c$ is the mass fraction at the chemical equilibrium, which is calculated from the density and temperature (or, the dynamic pressure and free stream enthalpy). Note that wall enthalpy $h_{w}$ is lower than free stream enthalpy $h$.

The nose radius for the sphere-shaped and blunt-body vehicles is normally adopted as parameter $R$ in Eqs. (6) and (7). In contrast, other shape parameter should be used for the inflatable reentry vehicle because this type of a vehicle has a relatively large membrane aeroshell and torus compared with the nose radius of the capsule. In this study, the shape parameter, $R$, is expressed as the geometric mean of nose radius of the capsule $R_{\mathrm{c}}$ and total length $D$, i.e.,

$$
R \rightarrow R_{\text {eff }}=R_{\mathrm{c}}^{0.7} D^{0.3}
$$

The heating-rate methods as expressed in Eqs. (6) and (7) are formulated under a chemical equilibrium flow assumption. Thus, the methods predict an inaccurate heat flux for the nonequilibrium flow problem under the NCW condition, which occurs in the present Titans vehicle reentry cases. Therefore, we propose the following heating-rate method for the nonequilibrium flows around an inflatable reentry vehicle:

$$
q_{\mathrm{c}, \text { neq }} \sqrt{\frac{R}{p_{0}}}=K\left(h-h_{w}\right)\left\{f\left(1-C_{n e q} \beta_{w}\right)+(1-f)\left(1-\frac{c \Delta h^{0}}{h}\right)\right\},
$$

where $K=6.62 \times 10^{-4} \mathrm{~W} /\left(\mathrm{m}^{3 / 2} \mathrm{~Pa}^{1 / 2} \mathrm{~J} / \mathrm{kg}\right)$ and $C_{\mathrm{neq}}=1.9$. The degree of dissociation at the stagnation point is simply modeled based on the present CFD results as

$$
\beta_{w}=A_{\mathrm{dod}} \exp \left(-\frac{C_{\mathrm{dod}}}{\rho_{\infty} U_{\infty} D}\right)
$$

where $A_{\text {dod }}=0.30$ and $C_{\text {dod }}=0.035$. Finally, blending function $f$ is given by

$$
f=\frac{1}{\pi} \arctan \left\{C_{\mathrm{b}}\left(h-h_{\mathrm{b}}\right)\right\}+\frac{1}{2},
$$

where $C_{\mathrm{b}}=10^{-5}$ and $h_{\mathrm{b}}=10^{7} \mathrm{~J} / \mathrm{kg}$.

A comparison of the heat flux on the stagnation point versus the flow enthalpy of the free stream under the NCW and FCW conditions in the CFD simulation is shown in Figs. 12(a) and 12(b), respectively. The heating-rate method for the FCW conditions calculated by Eq. (6) reproduces the tendency of the CFD results for the FCW conditions well, although there are slight discrepancies between the two methods. In addition, the results from the heating-rate method for a nonequilibrium flow under the NCW condition calculated by Eq. 


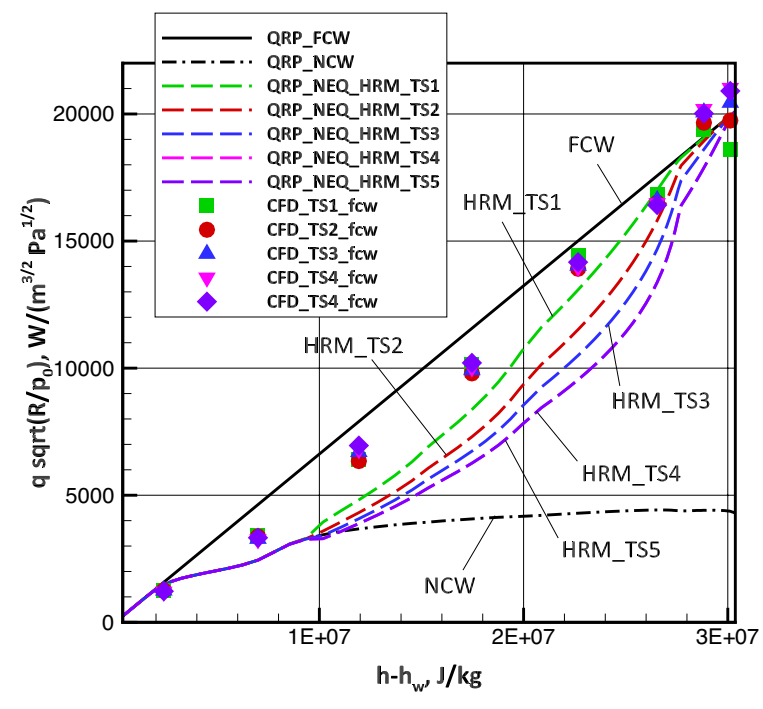

(a) Fully catalytic wall condition

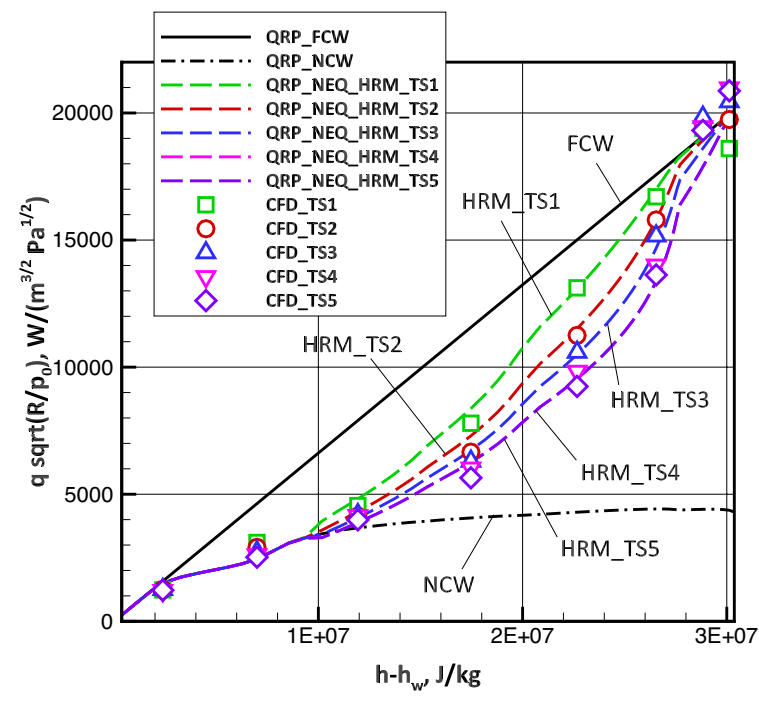

(b) Non-catalytic wall condition.

Figure 12: Comparisons of the relation between the heat flux on the stagnation point and freestream enthalpy for the TS1, TS2, TS3, TS4, and TS5 cases.

(9) show good agreement with the CFD results for the NCW condition (also see Appendix A). Because the flow fields around the Titans vehicle are strongly in nonequilibrium, the heating-rate method for the NCW condition given by $\mathrm{Eq}(7)$ is inaccurate under a higher enthalpy condition, i.e., higher altitude.

The heat fluxes on the torus and membrane for all the cases under the NCW and FCW conditions, respectively, are shown in Figs. 13(a) and 13(b). The heat fluxes are normalized using the stagnation heat flux, nose radius of the capsule, and total length, as follows:

$$
\begin{aligned}
& \bar{q}_{t}=\frac{q_{t}}{q_{c}} \sqrt{\frac{D}{R_{c}}}, \\
& \bar{q}_{m}=\frac{q_{m}}{q_{c}} \sqrt{\frac{D}{R_{c}}} .
\end{aligned}
$$

The normalized heat fluxes on the torus and membrane under the FCW condition have constant values of approximately 1.8 and 1.1, respectively. In contrast, under the NCW condition, slight discrepancies in the heat fluxes at higher and lower altitudes are confirmed.

\section{Conclusions}

The aerodynamic heating of an inflatable reentry vehicle (Titans) during reentry into the Earth's atmosphere by a controlled deorbit was investigated using the computational fluid dynamics approach, which was coupled with the structure analysis of its aeroshell composed of a thin-flare membrane.

The fundamental structures of the high-enthalpy nonequilibrium flow around the Titans vehicle were clarified. By parametric study varying the shape parameters of the Titans vehicle, surface catalysis, and turbulent model, the heat flux profile tendencies on the surface of the vehicle for these parameters were obtained. It was found that the heat flux on the 


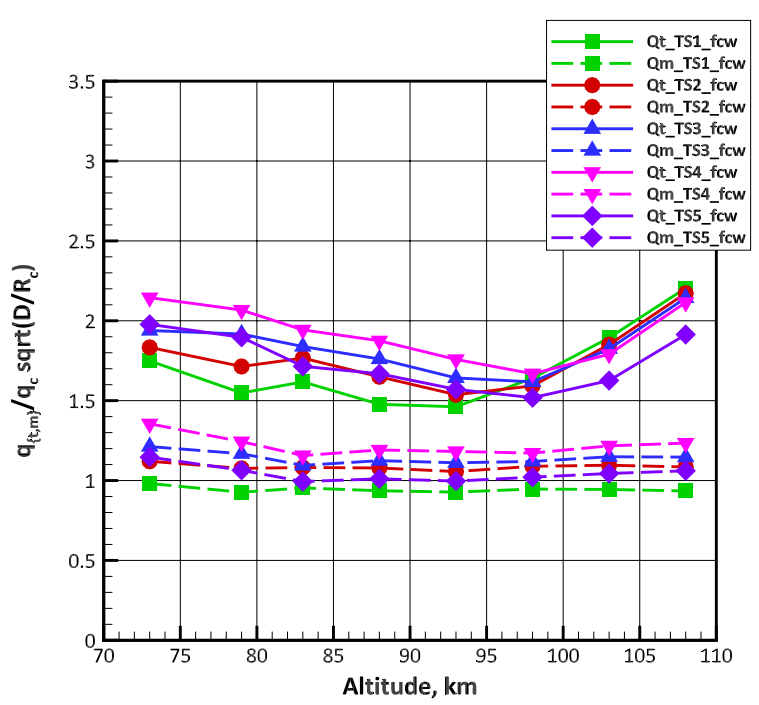

(a) Fully catalytic wall condition

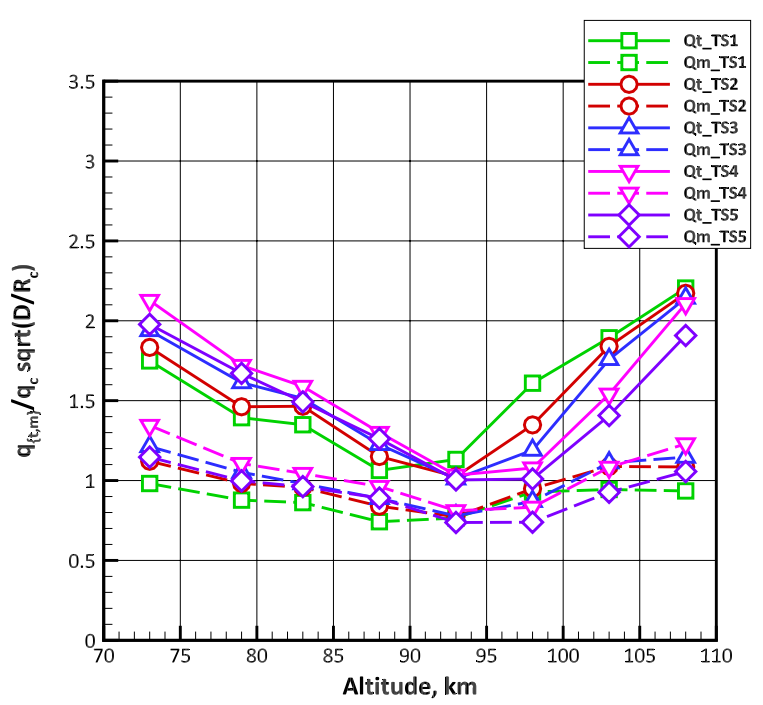

(b) Non-catalytic wall condition.

Figure 13: Heat fluxes on the torus and membrane for the TS1, TS2, TS3, TS4, and TS5 cases.

surface was strongly affected by the vehicle shape. The flare angle of the membrane aeroshell and membrane deformation had significant effects on the stagnation heat flux, but they barely affected the heat fluxes on the membrane and inflatable torus. In contrast, the surface catalysis and turbulence increased the heat flux on the entire surface of the vehicle.

Based on the present numerical simulation results, a new heating-rate method was proposed for a simple heat flux prediction for the nonequilibrium flows that tend to occur around the inflatable reentry vehicle. The nonequilibrium heating-rate method well-reproduced the stagnation heat fluxes from the numerical simulation results. In addition, the relation between heat flux on the membrane and torus was clarified.

\section{Appendix A: Heat Fluxes by Heating-Rate Method}

A comparison of the heat flux profiles at the stagnation calculated by the present heating-rate method and the CFD results for the FCW and NCW conditions versus the altitude is shown in Fig. 14. The heating-rate method for the nonequilibrium flows expressed by Eq. (9) reproduces the heat flux profiles under the NCW condition obtained by the CFD approach.

\section{Appendix B: Comparison with the Fay-Riddell formula}

The Fay-Riddell formula [28] is widely used to evaluate a stagnation heat flux of atmospheric reentry capsule, which is expressed for an axisymmetric nose as

$$
q_{\mathrm{c}, \mathrm{FR}}=0.763 \operatorname{Pr}_{w}^{-0.6}\left(\rho_{w} \mu_{w}\right)^{0.1}\left(\rho_{e} \mu_{e}\right)^{0.4}\left[1+\left(L e^{a}-1\right) \frac{h_{\mathrm{D}}}{h_{e}}\right]\left[h_{e}-h_{w}\right] \sqrt{\frac{d u_{e}}{d x}},
$$

where $\operatorname{Pr}_{w}$ and $L e$ are the Prandtl number set to 0.71 and Lewis number that is approximately unity. The dissociation energy, $h_{\mathrm{D}}$, of the freestream air is given by $c \Delta h^{0}$. The constant, " $a$," 


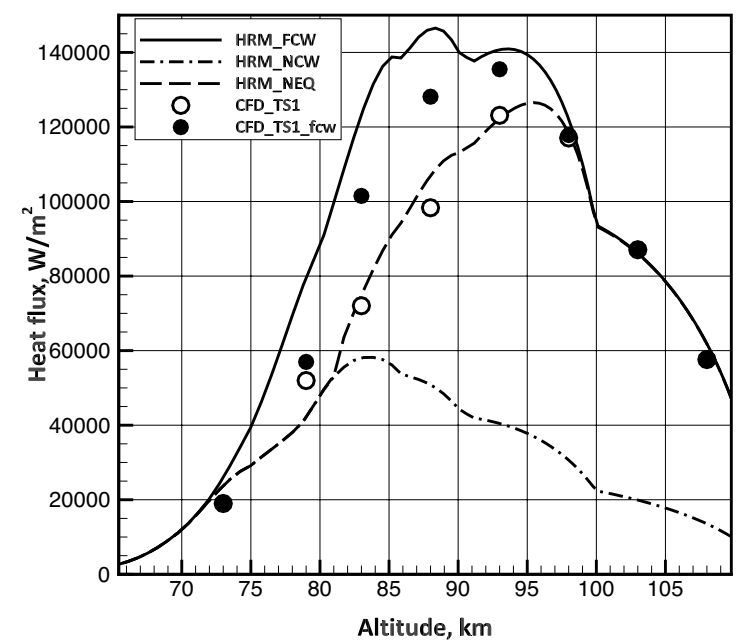

(a) "TS1"

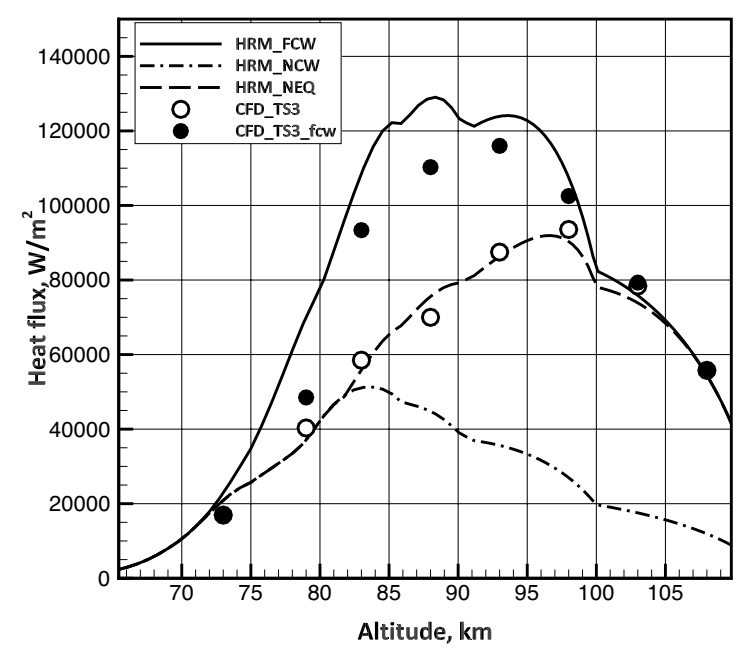

(c) "TS3"

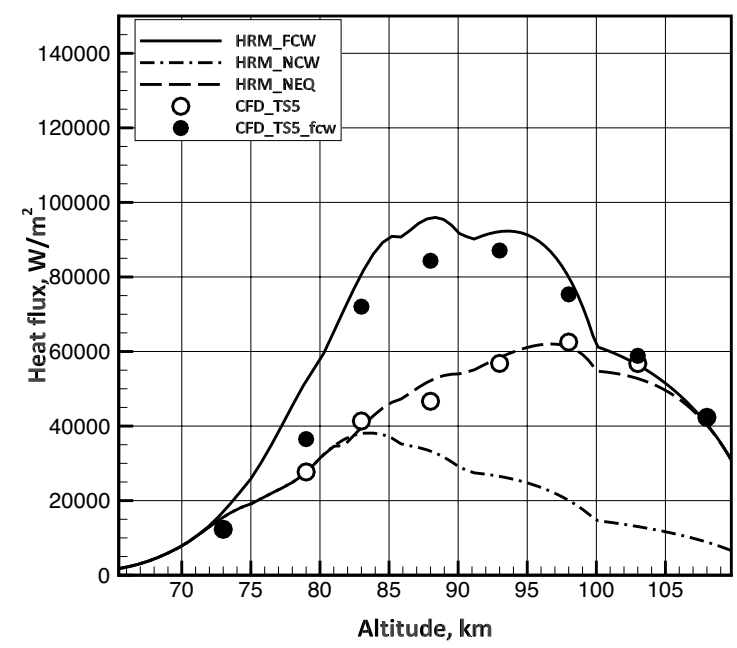

(e) "TS5"

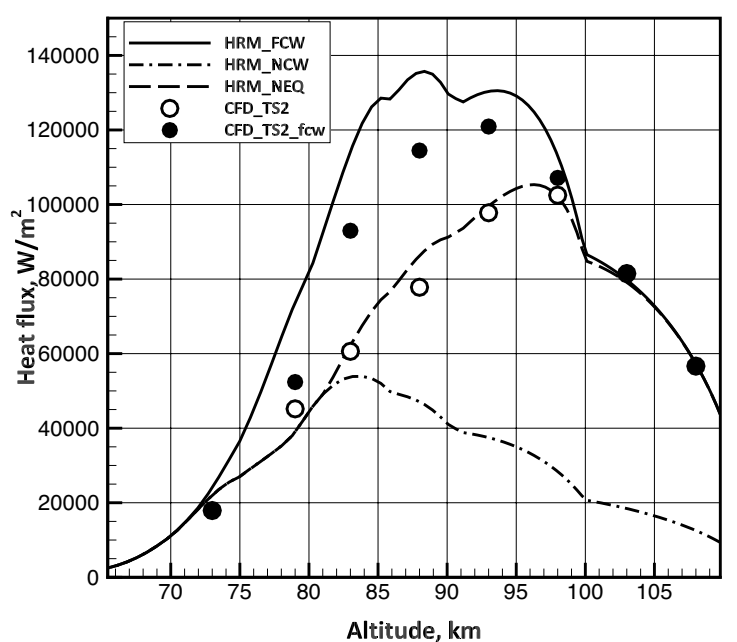

(b) "TS2"

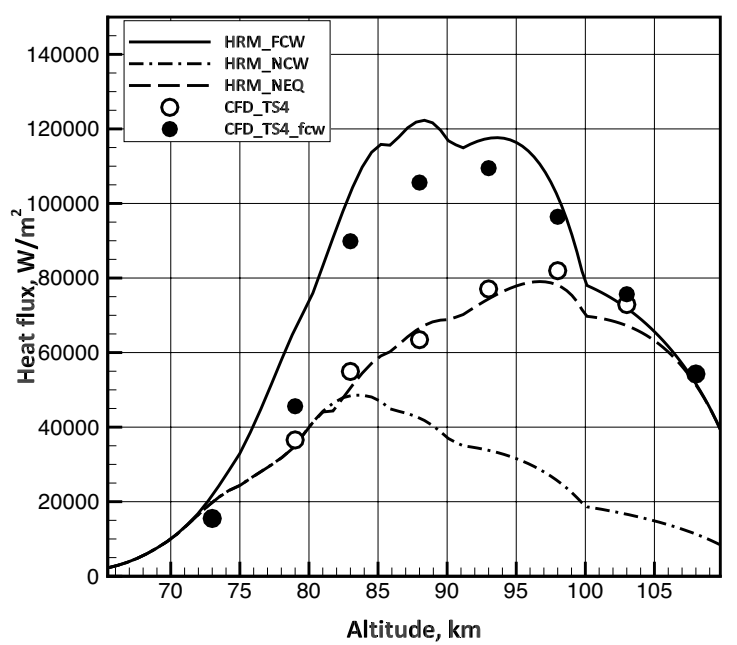

(d) "TS4"

Figure 14: Heat fluxes at the stagnation for the TS1, TS2, TS3, TS4, and TS5 cases. 
is 0.52 for an equilibrium boundary layer and 0.63 for a frozen boundary layer. Moreover,

$$
\frac{d u_{e}}{d x}=\frac{1}{R} \sqrt{\frac{2\left(p_{e}-p_{\infty}\right)}{\rho_{e}}} .
$$

The flow properties at the edge of the boundary layer can be calculated by the RankineHugoniot relations and isentropic flow relations. The viscosities at the edge and wall are calculated by the Sutherland formula. In addition, the density at the wall can be obtained, assuming that the pressure at the wall is equal to that at the edge of the boundary layer.

Figure 15 shows comparison of heat fluxes for the "TS2" case by the CFD, the heatingrate methods, and the Fay-Riddell formula changing the Lewis number and the constant " $a$." The nose radius, $R$, of Eq. (14) is set to the present geometric mean given by Eq. (8). The present heating-rate method reproduces the tendency that the Fay-Riddell formula does not reproduce for this case.

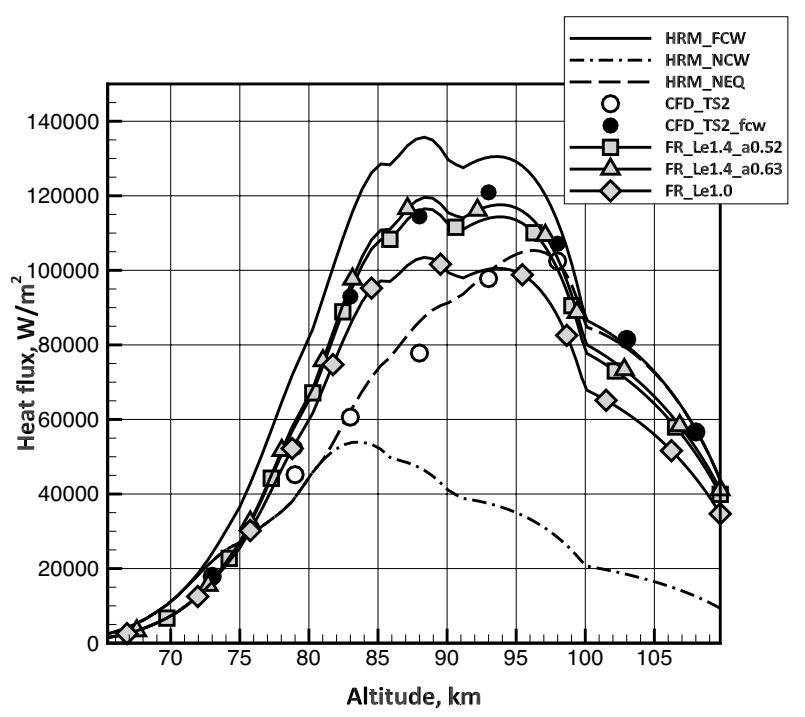

Figure 15: Comparison of heat fluxes on the stagnation for the baseline case "TS2".

\section{Appendix C: Simplified Heating-Rate Method}

To calculate the heat flux on stagnation points of the inflatable reentry vehicle from Eqs. (6), (7), and (9), it is convenient to use the following expressions:

$$
\begin{gathered}
q_{\mathrm{c}, \mathrm{fcw}}=K\left(h-h_{w}\right) \sqrt{\frac{p_{0}}{R}}, \\
q_{\mathrm{c}, \mathrm{ncw}}=q_{\mathrm{c}, \mathrm{fcw}}\left(1-\frac{c \Delta h^{0}}{h}\right), \\
q_{\mathrm{c}, \text { neq }}=q_{\mathrm{c}, \mathrm{fcw}}\left(1-C_{n e q} \beta_{\mathrm{w}}\right) f+q_{\mathrm{c}, \mathrm{ncw}}(1-f) .
\end{gathered}
$$


Table 3: Enthalpy of formation.

\begin{tabular}{c|c}
\hline \hline Species & $\Delta h_{s}^{0}(\mathrm{~J} / \mathrm{kg})$ \\
\hline $\mathrm{N}_{2}$ & 0 \\
$\mathrm{O}_{2}$ & 0 \\
$\mathrm{NO}$ & $2.995 \times 10^{6}$ \\
$\mathrm{~N}_{2}^{+}$ & $5.372 \times 10^{7}$ \\
$\mathrm{O}_{2}^{+}$ & $3.639 \times 10^{7}$ \\
$\mathrm{NO}^{+}$ & $3.282 \times 10^{7}$ \\
$\mathrm{~N}$ & $3.364 \times 10^{7}$ \\
$\mathrm{O}$ & $1.543 \times 10^{7}$ \\
$\mathrm{~N}^{+}$ & $1.339 \times 10^{8}$ \\
$\mathrm{O}^{+}$ & $9.787 \times 10^{7}$ \\
$\mathrm{e}^{-}$ & 0 \\
\hline \hline
\end{tabular}

Mass fraction, $c$, at the chemical equilibrium in Eq. (17) can be calculated according to Prabhu's work [29].

Alternatively, Eq. (18) can be replaced without blending function $f$ by the following expression:

$$
q_{\mathrm{c}, \text { neq }}=\min \left\{q_{\mathrm{c}, \mathrm{fcw}}, \max \left[q_{\mathrm{c}, \mathrm{fcw}}\left(1-C_{n e q} \beta_{\mathrm{w}}\right), q_{\mathrm{c}, \mathrm{ncw}}\right]\right\}
$$

The dynamic pressure and flow enthalpy of the free stream can be approximated as $p_{0}=\frac{1}{2} \rho_{\infty} U_{\infty}^{2}$ and $h=\frac{1}{2} U_{\infty}^{2}$, respectively. In addition, the wall enthalpy is generally lower than the flow enthalpy. Using these relations, Eq. (16) can be simplified as

$$
q_{\mathrm{c}, \mathrm{fcw}}=K^{\prime} U_{\infty}^{3} \sqrt{\frac{\rho_{\infty}}{R}}
$$

where $K^{\prime}=2.34 \times 10^{-4} \mathrm{~W} /\left(\mathrm{m}^{3 / 2} \mathrm{~Pa}^{1 / 2} \mathrm{~J} / \mathrm{kg}\right)$.

Enthalpy of formation $\Delta h_{s}^{0}$ for chemical species are listed in Table 3.

\section{Acknowledgments}

This work was supported by JSPS KAKENHI grant number $15 \mathrm{H} 04205$. The computations were performed using the computational facilities at the Research Institute for Information Technology, Kyushu University.

\section{References}

[1] T. Abe. "A Self-Consistent Tension Shell Structure for Application to Aerobraking Vehicle and Its Aerodynamic Characteristics". AIAA Paper 1988-3405, 1988.

[2] I.G. Clark, A.L. Hutchings, C.L. Tanner, and R.D. Braun. "Supersonic Inflatable Aerodynamic Decelerators for Use on Future Robotic Missions to Mars". Journal of Spacecraft and Rockets, 46(2):340-352, March - April 2009. 
[3] K. Yamada, D. Akita, E. Sato, K. Suzuki, T. Narumi, and T. Abe. "Flare-Type Membrane Aeroshell Flight Test at Free Drop from a Balloon". Journal of Spacecraft and Rockets, 46(3):606-614, May-June 2009.

[4] K. Yamada, M. Koyama, Y. Kimura, K. Suzuki, T. Abe, and A.K. Hayashi. "Hypersonic Wind Tunnel Test of a Flare-type Membrane Aeroshell for Atmospheric Entry Capsule". ISTS Special Issue: Selected papers from the 27th ISTS, Transactions of JSASS, 7(ists27):27-32, 2010.

[5] K. Yamada, T. Sonoda, K. Nakashino, and T. Abe. "Structural Strength of Flare-type Membrane Aeroshell Supported by Inflatable Tours against Aerodynamic Force". In Proceedings of 28th International Symposium on Space Technology and Science, ISTS 2011-c-34, Okinawa, Japan, June 5 - 122011.

[6] K. Yamada, Y. Nagata, T. Abe, K. Suzuki, O. Imamura, and D. Akita. "Suborbital Reentry Demonstration of Inflatable Flare-Type Thin-Membrane Aeroshell Using a Sounding Rocket". Journal of Spacecraft and Rockets, 52(1):275-284, February-March 2015.

[7] Y. Nagata, K. Yamada, T. Abe, and k Suzuki. "Attitude Dynamics for Flare-type Membrane Aeroshell Capsule in Reentry Flight Experiment". AIAA Paper 2013-1285, 2013.

[8] Y. Takahashi, K. Yamada, T. Abe, and K. Suzuki. "Aerodynamic Heating around Flaretype Membrane Inflatable Vehicle in Suborbital Reentry Demonstration Flight". Journal of Spacecraft and Rockets, 52(6):1530-1541, November 2015.

[9] K. Yamada, T. Moriyoshi, K. Matsumaru, H. Kanemaru, T. Araya, K. Suzuki5, O. Imamura, D. Akita, Y. Nagata, Y. Shoji, Y. Takahashi, Y. Watanabe, and T. Abe. "Reentry Nano-Satellite with Gossamer Aeroshell and GPS/Iridium Deployed from ISS". In Proceedings of 31th International Symposium on Space Technology and Science, ISTS 2017-f-021, Matsuyama, Ehime, Japan, June 3 - 92017.

[10] K. Yamada, K. Suzuki, T. Abe, O. Imamura, D. Akita, Y. Nagata, and Y. Takahashi. "Development of Flare-type Inflatable Membrane Aeroshell for Reentry Demonstration from LEO". AIAA Paper 2012-1016, 2015.

[11] Y. Takahashi, K. Yamada, and T. Abe. "Examination of Radio Frequency Blackout for an Inflatable Vehicle during Atmospheric Reentry". Journal of Spacecraft and Rockets, 51(2):430-441, March 2014.

[12] Y. Takahashi, D. Ha, N. Oshima, K. Yamada, T. Abe, and K. Suzuki. "Aerodecelerator Performance of Flare-Type Membrane Inflatable Vehicle in Suborbital Reentry". Journal of Spacecraft and Rockets, 54(5):993-1004, 2017.

[13] J. Guo, G. Lin, X. Bu, S. Fu, and Y. Chao. "Effect of static shape deformation on aerodynamics and aerothermodynamics of hypersonic inflatable aerodynamic decelerator". Acta Astronautica, 136:421-433, 2017.

[14] Y. Takahashi and K. Yamada. "Aerodynamic-Heating Analysis of Sample-Return Capsule in Future Trojan-Asteroid Exploration". Journal of Thermophysics and Heat Transfer, 2016. 
[15] J.M. Yos. "Transport Properties of Nitrogen, Hydrogen Oxygen and Air to 30,000 K". TRAD-TM-63-7, Research and Advanced Development Division, AVCO Corp., 1963.

[16] R.N. Gupta, J.M. Yos, R.A. Thompson, and K.P. Lee. "A Review of Reaction Rates and Thermodynamic and Transport Properties for an 11-Species Air Model for Chemical and Thermal Nonequilibrium Calculations to 30000 K". NASA RP-1232, Aug. 1990.

[17] M. Fertig, A. Dohr, and H.H. Frühaufu. "Transport Coefficients for High-Temperature Nonequilibrium Air Flows". AIAA Paper 98-2937, 1998.

[18] M. Fertig, A. Dohr, and H.H. Frühauf. "Transport Coefficients for High-Temperature Nonequilibrium Air Flows". Journal of Thermophysics and Heat Transfer, 15(2):148156, April-June 2001.

[19] C.F. Curtiss and J.O. Hirschfelder. "Transport Properties of Multicomponent Gas Mixture". Journal of Chemical Physics, 17(6):550-555, June 1949.

[20] C. Park. "Assessment of a Two-Temperature Kinetic Model for Dissociating and Weakly Ionizing Nitrogen". Journal of Thermophysics and Heat Transfer, 2(1):8-16, Jan.-March 1988.

[21] C. Park. Nonequilibrium Hypersonic Aerothermodynamics. Wiley, New York, 1990.

[22] E. Shima and K. Kitamura. "Parameter-Free Simple Low-Dissipation AUSM-Family Scheme for All Speeds". AIAA Journal, 49(8):1693-1709, August 2011.

[23] A. Jameson and S. Yoon. "Lower-Upper Implicit Schemes with Multiple Grids for the Euler Equations". AIAA Journal, 25(7):929-935, July 1987.

[24] T.R.A. Bussing and E.M. Murman. "Finite-Volume Method for the Calculation of Compressible Chemically Reacting Flows". AIAA Journal, 26(9):1070-1078, 1988.

[25] F.R. Menter. "Two-Equation Eddy-Viscosity Turbulence Models for Engineering Applications". AIAA Journal, 32(8):1598-1605, August 1994.

[26] J.G. Marvin and R.B. Pope. "Laminar Convective Heating and Ablation in the Mars Atmosphere". AIAA Journal, 5(2):240-248, February 1967.

[27] R.B. Pope. "Measurements of Enthalpy in Low-Density Arc-Heated Flows". AIAA Journal, 6(1):103-110, January 1968.

[28] J.A. Fay and F.R. Riddell. "Theory of stagnation point heat transfer in dissociated air". Journal of the Aerospace Sciences, 25(2):73-85, February 1958.

[29] R.K. Prabhu and W.D. Erickson. "A rapid method for the computation of equilibrium chemical composition of air to $15000 \mathrm{~K}$ ". NASA TP-2792, 1988. 